\title{
ENERGY ESTIMATES FOR VARIATIONAL MINIMIZERS OF A PARABOLIC DOUBLY NONLINEAR EQUATION ON METRIC MEASURE SPACES
}

\author{
Per-Anders Ivert, Niko Marola and Mathias Masson \\ pa.ivert@gmail.com \\ University of Helsinki, Department of Mathematics and Statistics \\ P.O. Box 68, FI-00014 University of Helsinki, Finland; niko.marola@helsinki.fi \\ Aalto University, School of Science and Technology, Department of Mathematics \\ P.O. Box 11100, FI-00076 Aalto, Finland; mathias.masson.finland@gmail.com
}

\begin{abstract}
In this paper a variational approach is taken to study a doubly nonlinear parabolic equation. We consider energy estimates for parabolic minimizers related to this equation. These energy estimates play a fundamental role in obtaining Harnack estimates. Our treatment is done in general metric measure spaces with a doubling measure and a Poincaré inequality.
\end{abstract}

\section{Introduction}

This paper is a continuation to a recent paper [4]. We study parabolic minimizers which are related to the doubly nonlinear parabolic equation

$$
\frac{\partial\left(|u|^{p-2} u\right)}{\partial t}-\nabla \cdot\left(|\nabla u|^{p-2} \nabla u\right)=0, \quad 1<p<\infty .
$$

Our study is carried out in the context of a general complete metric measure space $(X, d, \mu)$ with a doubling measure $\mu$. We shall also assume that $X$ supports a weak Poincaré inequality [1].

A real-valued function $u$ on $\Omega \times(0, T)$, where $\Omega$ is a non-empty open set in $X$, is a parabolic minimizer if it satisfies the inequality

$$
p \int_{\operatorname{supp}(\phi)}|u|^{p-2} u \frac{\partial \phi}{\partial t} d \mu d t+\int_{\operatorname{supp}(\phi)} g_{u}^{p} d \mu d t \leq \int_{\operatorname{supp}(\phi)} g_{u+\phi}^{p} d \mu d t
$$

for every compactly supported $\phi \in \operatorname{Lip}(\Omega \times(0, T))$. Here we write $g_{u}$ for the minimal $p$-weak upper gradient of $u$ [1]. In the Euclidean case, we can take $g_{u}$ to be $|\nabla u|$ almost everywhere, and it can be shown that every weak solution to (1.1) is a parabolic minizer and, conversely, every parabolic minimizer is a weak solution of (1.1). Parabolic minimizers have been studied in [6] and [7]. The regularity theory for parabolic minizers on metric measure spaces has been considered in [3], [4], and [5].

In [4] it is shown, that if $X$ is a complete metric space equipped with a doubling measure $\mu$ such that the space supports also a weak Poincaré inequality, then a positive parabolic minimizer which is both bounded and bounded away from zero satisfies certain Caccioppoli type energy estimates and, moreover, Harnack estimates

doi:10.5186/aasfm.2014.3936

2010 Mathematics Subject Classification: Primary 35B45; Secondary 35K55, 30L99.

Key words: Doubling measure, energy estimates, Harnack inequality, parabolic minimizer, Poincaré inequality, subminimizer, superminimizer. 
hold if $X$ is assumed to be geodesic. In the present note, we remove these extra technical assumptions concerning the lower and upper bound of a positive minimizer. More precisely, we show that any positive parabolic minimizer $u$ satisfies the same fundamental energy estimates which are used in the article [4] as a basis for proving the Harnack inequality. The approach is purely variational and, in particular, does not base on equation (1.1).

More importantly, our technique for removing the extra assumptions bases on the dampening of the growth rate of powers of a minimizer. The traditional truncation argument is problematic in the context of parabolic (quasi)minimizers. Indeed, it is not known whether parabolic super- or subminimizers are produced by truncating minimizers. The dampening technique presented here circumvents this, and therefore, seems to be interesting in its own right and new.

Lastly, we mention that it seems feasible that the technical assumption $u \geq \rho>0$ in the estimates for weak super- and subsolutions to the doubly nonlinear equation in Kinnunen-Kuusi [2] can be removed by using the dampening technique presented in the present note.

Acknowledgements. Work partially done during the second author's visit to the Institut Mittag-Leffler (Djursholm, Sweden) in September-December, 2013. Support by the institute, by the Academy of Finland, and by the Väisälä Foundation are gratefully acknowledged.

\section{Energy estimates}

The notation and parabolic setting in this paper are as in [4]. We refrain from giving a detailed discussion on the metric measure space context (doubling condition, upper gradients, weak Poincaré inequalities etc.) and content ourselves with refering to [4] or for a detailed study to a recent monograph [1]. Our standing assumptions in this paper are the following. By the triplet $(X, d, \mu)$ we denote a complete metric space $X$, where $d$ is the metric and $\mu$ a Borel measure on $X$. The measure $\mu$ is supposed to be doubling. We also assume that $X$ supports a weak $(1, p)$-Poincaré inequality, where $1<p<\infty$. For any open set $\Omega$ of $X$ we write $\Omega_{T}=\Omega \times(0, T)$, for $T>0$, and the product measure $d \mu d t$ is written as $d \nu$. A minimal $p$-weak upper gradient of a real-valued function $u$ on $X$ is written as $g_{u}$. The reader not familiar with the metric space set-up may consider $g_{u}$ to be the modulus of the distributional gradient $|\nabla u|$ and the Newtonian space $N^{1, p}$ to be the classical Sobolev space $W^{1, p}$.

We say that $u \in L_{\text {loc }}^{p}\left(0, T ; N_{\text {loc }}^{1, p}(\Omega)\right)$ is a parabolic minimizer related to the doubly nonlinear equation if the inequality

$$
p \int_{\operatorname{supp}(\phi)}|u|^{p-2} u \frac{\partial \phi}{\partial t} d \nu+\int_{\operatorname{supp}(\phi)} g_{u}^{p} d \nu \leq \int_{\operatorname{supp}(\phi)} g_{u+\phi}^{p} d \nu,
$$

holds for all $\phi \in \operatorname{Lip}_{0}\left(\Omega_{T}\right)=\left\{f \in \operatorname{Lip}\left(\Omega_{T}\right): \operatorname{supp}(f) \subset \Omega_{T}\right\}$. If (2.1) holds for all nonnegative $\phi \in \operatorname{Lip}_{0}\left(\Omega_{T}\right)$ a function $u \in L_{\mathrm{loc}}^{p}\left(0, T ; N_{\mathrm{loc}}^{1, p}(\Omega)\right)$ is a parabolic superminimizer; and a parabolic subminimizer if (2.1) holds for all nonpositive $\phi \in \operatorname{Lip}_{0}\left(\Omega_{T}\right)$.

Obtaining energy estimates for parabolic minimizers is based on testing $u$ with a suitably chosen test function which depends on $u$ itself, and then performing various basic techniques from analysis to obtain an estimate of the desired form. One of these techniques is partial integration with respect to time. In order to be able to do 
this later on, we use a certain time regularization procedure to alter inequality (2.1) to a more convenient form for our purposes.

Let $u$ be a nonnegative parabolic (sub- or super)minimizer, and let $\phi \in \operatorname{Lip}_{0}\left(\Omega_{T}\right)$ be a valid test function. For sufficiently small $\delta>0$, we take a standard mollifier $\xi_{\delta} \in C_{0}^{\infty}(\mathbf{R})$ satisfying $\operatorname{supp}\left(\xi_{\delta}\right) \subset[-\delta, \delta], \xi_{\delta} \geq 0$, and $\left\|\xi_{\delta}\right\|_{L^{1}(\mathbb{R})}=1$, and denote $(\phi)_{\delta}(x, t):=\int_{-\delta}^{\delta} \phi(x, t-s) \xi_{\delta}(s) d s$ in $\Omega_{T}$.

We test $u$ with $\phi(x, t+s)$, where $s$ is small enough. By (2.1), after conducting a change of variable in the first integral on the left hand side, then multiplying both sides of the obtained inequality with the standard mollifyer $\xi_{\delta}(s)$, and integrating the resulting expression with respect to $s$ gives us, after using Fubini's theorem, the inequality

$$
p \int_{\operatorname{supp}(\phi)}\left(u^{p-1}\right)_{\delta} \frac{\partial \phi}{\partial t} d \nu+\int_{\operatorname{supp}(\phi)}\left(g_{u}^{p}\right)_{\delta} d \nu \leq \int_{\operatorname{supp}(\phi)}\left(g_{u(x, t-s)+\phi(x, t)}^{p}\right)_{\delta} d \nu
$$

As will be seen below, this inequality is now in a more convenient form for establishing energy estimates.

Strictly speaking, in order to use Fubini's theorem above, one needs to know that the parabolic minimal $p$-weak upper gradient $g_{u}(x, t)$ of a parabolic minimizer $u$ is measurable on $\Omega_{T}$ with respect to the product measure $\nu$. In the metric measure space case it seems uncertain if one can prove this. In the Euclidean space product measurablility is known, and hence the difficulty is not present. Also, in the metric space setting one can bypass a use of mollification and Fubini's theorem by assuming more time regularity on a parabolic minimizer $u$. Since in this paper we do not make any additional assumptions on the time regularity of $u$ and, on the other hand, the underlying space is a general metric measure space, the reader should keep in mind that in our treatment the difficulty related to the product measurability is in fact omitted.

We shall next conduct further general preparative work for establishing energy estimates, by studying how a cutoff in time affects the estimate in (2.2).

We replace in $(2.2)$ the test function $\phi \in \operatorname{Lip}_{0}\left(\Omega_{T}\right)$ with the function

$$
\phi(x, t)\left(\eta_{h, \sigma}(t)-\eta_{h, \tau}(t)\right) \in \operatorname{Lip}_{0}\left(\Omega_{T}\right),
$$

where $0<\sigma<\tau<T$ and the function $\eta_{h, \tau}$ is the piecewise linear cutoff function

$$
\eta_{h, \tau}(t)= \begin{cases}0 & \text { if } 0<t \leq \tau \\ (t-\tau) / h & \text { if } \tau<t<\tau+h \\ 1 & \text { if } \tau+h \leq t \leq T\end{cases}
$$

After letting $h \rightarrow 0+$, we obtain by Lebesgue's differentiation theorem and by integrating by parts

$$
-p \int_{\sigma}^{\tau} \int_{\Omega} \frac{\partial\left(u^{p-1}\right)_{\delta}}{\partial t} \phi d \mu d t+\int_{\sigma}^{\tau} \int_{\Omega}\left(g_{u}^{p}\right)_{\delta} d \mu d t \leq \int_{\sigma}^{\tau} \int_{\Omega}\left(g_{u(x, t-s)+\phi(x, t)}^{p}\right)_{\delta} d \mu d t
$$

for every valid test function $\phi \in \operatorname{Lip}_{0}\left(\Omega_{T}\right)$ and almost every $0<\sigma<\tau<T$.

We are now ready to prove the energy estimates for parabolic super- and subminimizers. It is not obvious to see how the interplay between the time regularization and the dampening function $H_{\lambda}(u)$ defined below should be organized for parabolic minimizers related to the doubly nonlinear equation. That is why instead of presenting only a formal proof, we also take into account the time regularization. 
We begin by proving the estimate for positive superminimizers; cp. Lemma 3.1 in [4].

Lemma 2.1. Let $u$ be a positive parabolic superminimizer and $0<\varepsilon \neq p-1$. Then

$$
\begin{aligned}
& \underset{0<t<T}{\operatorname{ess} \sup } \int_{\Omega} u^{p-1-\varepsilon} \varphi^{p} d \mu+\int_{\operatorname{supp}(\varphi)} g_{u}^{p} u^{-1-\varepsilon} \varphi^{p} d \nu \\
& \leq\left(1+\frac{p}{\varepsilon}\right)\left(C_{1} \int_{\operatorname{supp}(\varphi)} u^{p-1-\varepsilon} g_{\varphi}^{p} d \nu+C_{2} \int_{\operatorname{supp}(\varphi)} u^{p-1-\varepsilon}\left|\left(\varphi^{p}\right)_{t}\right| d \nu\right)
\end{aligned}
$$

for every $\varphi \in \operatorname{Lip}_{0}\left(\Omega_{T}\right), 0 \leq \varphi \leq 1$, where

$$
C_{1}=\left(\frac{p}{\varepsilon}\right)^{p-1} \quad \text { and } \quad C_{2}=\frac{p-1}{|p-1-\varepsilon|} .
$$

Proof. For $\lambda>0$ we define the function $H_{\lambda}(s)$ for nonnegative $s$ by setting

$$
H_{\lambda}(s)= \begin{cases}\lambda^{-\varepsilon}+\varepsilon \lambda^{-\varepsilon-1}(\lambda-s) & \text { if } 0 \leq s \leq \lambda, \\ s^{-\varepsilon} & \text { if } s>\lambda .\end{cases}
$$

Then $H_{\lambda}$ is continuously differentiable, and

$$
H_{\lambda}^{\prime}(s)= \begin{cases}-\varepsilon \lambda^{-\varepsilon-1} & \text { if } 0 \leq s \leq \lambda, \\ -\varepsilon s^{-\varepsilon-1} & \text { if } s>\lambda .\end{cases}
$$

We note that $\left|H^{\prime}(s)\right| \leq \varepsilon \lambda^{-\varepsilon-1}$. We set $\alpha=\frac{1}{\varepsilon} \lambda^{\varepsilon+1}$, and so $\alpha\left|H^{\prime}(s)\right| \leq 1$ for all $s \geq 0$. Moreover, in order to be handle the time regularization below, we define $\widetilde{H_{\lambda}}(s)=H_{\lambda}\left(s^{1 /(p-1)}\right)$. We also define the function $h_{\lambda}(s)$ for $s \geq 0$ by setting

$$
h_{\lambda}(s)= \begin{cases}\int_{0}^{s} \widetilde{H_{\lambda}}(r) d r & \text { if } \varepsilon<p-1, \\ \int_{s}^{\infty} \widetilde{H_{\lambda}}(r) d r & \text { if } \varepsilon>p-1\end{cases}
$$

and let $\iota=\operatorname{sgn}(p-1-\varepsilon)$.

Let $\varphi$ be a function in $\operatorname{Lip}_{0}\left(\Omega_{T}\right)$ such that $0 \leq \varphi \leq 1$. We choose the nonnegative function $\phi=\alpha \widetilde{H}_{\lambda}\left(\left(u^{p-1}\right)_{\delta}\right) \varphi^{p}$ in (2.3), and note that defined this way $\phi$ is a valid test function. The first term on the left hand side of (2.3) becomes, after integrating by parts

$$
\begin{aligned}
& -p \int_{\tau_{1}}^{\tau_{2}} \int_{\Omega} \frac{\partial\left(u^{p-1}\right)_{\delta}}{\partial t} \phi d \mu d t=-\iota \alpha p \int_{\tau_{1}}^{\tau_{2}} \int_{\Omega} \frac{\partial h_{\lambda}\left(\left(u^{p-1}\right)_{\delta}\right)}{\partial t} \varphi^{p} d \mu d t \\
& =-\iota \alpha p\left[\int_{\Omega \times\{t\}} h_{\lambda}\left(\left(u^{p-1}\right)_{\delta}\right) \varphi^{p} d \mu\right]_{t=\tau_{1}}^{\tau_{2}}+\iota \alpha p \int_{\tau_{1}}^{\tau_{2}} \int_{\Omega} h_{\lambda}\left(\left(u^{p-1}\right)_{\delta}\right)\left(\varphi^{p}\right)_{t} d \mu d t .
\end{aligned}
$$

Letting then $\delta \rightarrow 0$, it can shown by Fubini's theorem and the properties of time mollifications that

$$
\begin{aligned}
& \lim _{\delta \rightarrow 0}\left|\iota \alpha p \int_{\tau_{1}}^{\tau_{2}} \int_{\Omega} h_{\lambda}\left(\left(u^{p-1}\right)_{\delta}\right)\left(\varphi^{p}\right)_{t} d \mu d t\right| \leq \alpha p \int_{\tau_{1}}^{\tau_{2}} \int_{\Omega}\left|h_{\lambda}\left(u^{p-1}\right)\right|\left|\left(\varphi^{p}\right)_{t}\right| d \mu d t \\
& \lim _{\delta \rightarrow 0}\left(-\iota \alpha p\left[\int_{\Omega \times\{t\}} h_{\lambda}\left(\left(u^{p-1}\right)_{\delta}\right) \varphi^{p} d \mu\right]_{t=\tau_{1}}^{\tau_{2}}\right)=-\iota \alpha p\left[\int_{\Omega \times\{t\}} h_{\lambda}\left(u^{p-1}\right) \varphi^{p} d \mu\right]_{t=\tau_{1}}^{\tau_{2}} .
\end{aligned}
$$


For the upper gradient terms in (2.3) we have

$$
\lim _{\delta \rightarrow 0} \int_{\tau_{1}}^{\tau_{2}} \int_{\Omega}\left(g_{u}^{p}\right)_{\delta} d \mu d t=\int_{\tau_{1}}^{\tau_{2}} \int_{\Omega} g_{u}^{p} d \mu d t
$$

and

$$
\begin{aligned}
& \limsup _{\delta \rightarrow 0} \int_{\tau_{1}}^{\tau_{2}} \int_{\Omega}\left(g_{u(\cdot,-s)+\alpha \widetilde{H_{\lambda}}\left(\left(u^{p-1}\right)_{\delta}\right) \varphi^{p}}^{p}\right)_{\delta} d \mu d t \\
& \leq \int_{\tau_{1}}^{\tau_{2}} \int_{\Omega} g_{u+\alpha \widetilde{H_{\lambda}}\left(u^{p-1}\right) \varphi^{p}}^{p} d \mu d t=\int_{\tau_{1}}^{\tau_{2}} \int_{\Omega} g_{u+\alpha H_{\lambda}(u) \varphi^{p}}^{p} d \mu d t .
\end{aligned}
$$

To verify that the inequality in (2.5) holds, we refer to [5, Section 6]. In order not to stray away from our main focus, we omit the proof. Combining the above results into (2.3), we obtain the inequality

$$
\begin{aligned}
& -\iota \alpha p\left[\int_{\Omega \times\{t\}} h_{\lambda}\left(u^{p-1}\right) \varphi^{p} d \mu\right]_{t=\tau_{1}}^{\tau_{2}}+\int_{\tau_{1}}^{\tau_{2}} \int_{\Omega} g_{u}^{p} d \mu d t \\
& \leq \int_{\tau_{1}}^{\tau_{2}} \int_{\Omega} g_{u+\alpha H_{\lambda}(u) \varphi^{p}}^{p} d \mu d t+\alpha p \int_{\tau_{1}}^{\tau_{2}} \int_{\Omega}\left|h_{\lambda}\left(u^{p-1}\right)\right|\left|\left(\varphi^{p}\right)_{t}\right| d \mu d t .
\end{aligned}
$$

for almost every $0<\tau_{1}<\tau_{2}<T$. Issues related to time regularity have now been dealt with, and we can continue proving the energy estimate. We begin by observing that

$$
g_{u+\alpha H_{\lambda}(u) \varphi^{p}} \leq\left(1+\alpha H_{\lambda}^{\prime}(u) \varphi^{p}\right) g_{u}+\alpha p \varphi^{p-1} H_{\lambda}(u) g_{\varphi} .
$$

That (2.7) holds is not evident, but since $\left|\alpha H_{\lambda}^{\prime}(u)\right|<1$, the inequality can be shown to be true using the pathwise properties of upper gradients, completely analogously to what is done in the proof of Lemma 3.1 in [4]. The inequality in (2.7) can be rewritten as

$$
g_{u+\alpha H_{\lambda}(u) \varphi^{p}} \leq\left(1-\alpha\left|H_{\lambda}^{\prime}(u)\right| \varphi^{p}\right) g_{u}+\alpha\left|H_{\lambda}^{\prime}(u)\right| \varphi^{p} \frac{p H_{\lambda}(u)}{\varphi\left|H_{\lambda}^{\prime}(u)\right|} g_{\varphi},
$$

and since $0 \leq \alpha\left|H_{\lambda}^{\prime}(u)\right| \varphi(x, t)^{p} \leq 1$, we get by the convexity of $t \mapsto t^{p}$ :

$$
g_{u+H_{\lambda}(u) \varphi^{p}}^{p} \leq\left(1-\alpha\left|H_{\lambda}^{\prime}(u)\right| \varphi^{p}\right) g_{u}^{p}+\alpha p^{p} \frac{H_{\lambda}(u)^{p}}{\left|H_{\lambda}^{\prime}(u)\right|^{p-1}} g_{\varphi}^{p} .
$$

Plugging this into (2.6) gives us

$$
\begin{aligned}
& -\iota \alpha p\left[\int_{\Omega \times\{t\}} h_{\lambda}\left(u^{p-1}\right) \varphi^{p} d \mu\right]_{t=\tau_{1}}^{\tau_{2}}+\alpha \int_{\tau_{1}}^{\tau_{2}} \int_{\Omega}\left|H_{\lambda}^{\prime}(u)\right| \varphi^{p} g_{u}^{p} d \mu d t \\
& \leq \alpha p^{p} \int_{\tau_{1}}^{\tau_{2}} \int_{\Omega} \frac{H_{\lambda}(u)^{p}}{\left|H_{\lambda}^{\prime}(u)\right|^{p-1}} g_{\varphi}^{p} d \mu d t+\alpha p \int_{\tau_{1}}^{\tau_{2}} \int_{\Omega}\left|h_{\lambda}\left(u^{p-1}\right)\right|\left|\left(\varphi^{p}\right)_{t}\right| d \mu d t .
\end{aligned}
$$

The inequality in (2.8) is independent of the scaling factor $\alpha$ so we can divide it out. Since the constants on the right hand side of the estimate (2.8) do not depend on the choice of $\tau$, the inequality (2.8) implies

$$
\begin{aligned}
& \underset{0<t<T}{\operatorname{ess} \sup } p \int_{\Omega} h_{\lambda}\left(u(x, t)^{p-1}\right) \varphi(x, t)^{p} d \mu \\
& \leq p^{p} \int_{\Omega_{T}} \frac{H_{\lambda}(u)^{p}}{\left|H_{\lambda}^{\prime}(u)\right|^{p-1}} g_{\varphi}^{p} d \nu+p \int_{\Omega_{T}}\left|h_{\lambda}\left(u^{p-1}\right)\right|\left|\left(\varphi^{p}\right)_{t}\right| d \nu .
\end{aligned}
$$


On the other hand, the inequality (2.8) gives us also

$$
\int_{\Omega_{T}}\left|H_{\lambda}^{\prime}(u)\right| \varphi^{p} g_{u}^{p} d \nu \leq p^{p} \int_{\Omega_{T}} \frac{H_{\lambda}(u)^{p}}{\left|H_{\lambda}^{\prime}(u)\right|^{p-1}} g_{\varphi}^{p} d \nu+p \int_{\Omega_{T}}\left|h_{\lambda}\left(u^{p-1}\right)\right|\left|\left(\varphi^{p}\right)_{t}\right| d \nu .
$$

Inequalities (2.9) and (2.10) are valid for all $0<\varepsilon \neq p-1$. Letting $\lambda$ decrease to 0 , we see that $H_{\lambda}(u),\left|H_{\lambda}^{\prime}(u)\right|$ and $h_{\lambda}(u)$ converge pointwise in a monotonously increasing way. In particular, we check by using a change of variable and then the theorem of dominated convergence that in the case $\varepsilon<p-1$

$$
\begin{aligned}
\lim _{\lambda \rightarrow 0} h_{\lambda}\left(u^{p-1}\right) & =\lim _{\lambda \rightarrow 0} \int_{0}^{u^{p-1}} H_{\lambda}\left(r^{\frac{1}{p-1}}\right) d r=\lim _{\lambda \rightarrow 0}(p-1) \int_{0}^{u} s^{p-2} H_{\lambda}(s) d s \\
& =(p-1) \int_{0}^{u} s^{p-2-\varepsilon} d s=\frac{p-1}{p-1-\varepsilon} u^{p-1-\varepsilon},
\end{aligned}
$$

and by a similar reasoning in the case $\varepsilon>p-1$

$$
\lim _{\lambda \rightarrow 0} h_{\lambda}\left(u^{p-1}\right)=-\frac{p-1}{p-1-\varepsilon} u^{p-1-\varepsilon} .
$$

Thus, by the monotone convergence theorem, we have

$$
\begin{aligned}
& \lim _{\lambda \rightarrow 0} \operatorname{ess\operatorname {sup}} p \int_{\Omega<t<T} h_{\lambda}\left(u^{p-1}\right) \varphi^{p} d \mu=\underset{0<t<T}{\operatorname{ess} \sup } \frac{p(p-1)}{|p-1-\varepsilon|} \int_{\Omega} u^{p-\varepsilon-1} \varphi^{p} d \mu, \\
& \lim _{\lambda \rightarrow 0} \int_{\Omega_{T}}\left|H_{\lambda}^{\prime}(u)\right| \varphi^{p} g_{u}^{p} d \nu=\varepsilon \int_{\Omega_{T}} g_{u}^{p} u^{-1-\varepsilon} \varphi^{p} d \nu, \\
& \lim _{\lambda \rightarrow 0} p \int_{\Omega_{T}}\left|h_{\lambda}\left(u^{p-1}\right)\right|\left|\left(\varphi^{p}\right)_{t}\right| d \nu=\frac{p(p-1)}{|p-1-\varepsilon|} \int_{\Omega_{T}} u^{p-1-\varepsilon}\left|\left(\varphi^{p}\right)_{t}\right| d \nu .
\end{aligned}
$$

It remains to verify the convergence of the last integral in (2.9) and (2.10) as $\lambda \rightarrow 0$. If the integral

$$
\int_{\operatorname{supp}(\phi)} u^{p-1-\varepsilon} g_{\phi}^{p} d \nu
$$

was infinite, then the estimate in (2.4) is trivially true and the proof is complete. Assume thus that the integral in (2.12) is finite. At the points $(x, t) \in \Omega_{T}$ where $0<u(x, t)<\lambda$, we have

$$
\frac{H_{\lambda}(u)^{p}}{\left|H_{\lambda}^{\prime}(u)\right|^{p-1}}= \begin{cases}\left(\varepsilon^{\frac{1}{p}-1} \lambda^{1-\frac{\varepsilon+1}{p}}+\varepsilon^{\frac{1}{p}}(\lambda-u) \lambda^{-\frac{\varepsilon+1}{p}}\right)^{p}, & 0<u(x, t)<\lambda, \\ \varepsilon^{1-p} u^{p-1-\varepsilon}, & u(x, t) \geq \lambda .\end{cases}
$$

From this expression we see by the theorem of dominated convergence that

$$
\lim _{\lambda \rightarrow 0} p^{p} \int_{\Omega_{T}} \frac{H_{\lambda}(u)^{p}}{\left|H_{\lambda}^{\prime}(u)\right|^{p-1}} g_{\varphi}^{p} d \nu=p^{p} \varepsilon^{1-p} \int_{\Omega_{T}} u^{p-1-\varepsilon} g_{\varphi}^{p} d \nu .
$$

Indeed, if $\varepsilon<p-1$ then we can use $H_{1}(u)^{p} /\left|H_{1}^{\prime}(u)\right|^{p-1} g_{\varphi}^{p}$ to dominate, and in the case in which $\varepsilon>p-1$ the term $\varepsilon^{1-p} u^{p-1-\varepsilon} g_{\varphi}^{p}$ dominates the integrand. Combining (2.11) with (2.13) through (2.9) and (2.10), and summing up the resulting inequalities completes the proof.

We then prove the energy estimate for positive subminimizers; cp. Lemma 4.1 in [4]. 
Lemma 2.2. Let $u$ be a nonnegative parabolic subminimizer and let $\varepsilon \geq 1$. Then

$$
\begin{aligned}
& \underset{0<t<T}{\operatorname{ess} \sup } \int_{\Omega} u^{p-1+\varepsilon} \varphi^{p} d \mu+\int_{\operatorname{supp}(\varphi)} g_{u}^{p} u^{-1+\varepsilon} \varphi^{p} d \nu \\
& \leq\left(1+\frac{p}{\varepsilon}\right)\left(C_{1} \int_{\operatorname{supp}(\varphi)} u^{p-1+\varepsilon} g_{\varphi}^{p} d \nu+C_{2} \int_{\operatorname{supp}(\varphi)} u^{p-1+\varepsilon}\left|\left(\varphi^{p}\right)_{t}\right| d \nu\right)
\end{aligned}
$$

for every $\varphi \in \operatorname{Lip}_{0}\left(\Omega_{T}\right), 0 \leq \varphi \leq 1$, where

$$
C_{1}=\left(\frac{p}{\varepsilon}\right)^{p-1} \quad \text { and } \quad C_{2}=\frac{p-1}{|p-1+\varepsilon|} .
$$

Proof. For $\lambda>0$ we define the function $H_{\lambda}(s)$ by setting

$$
H_{\lambda}(s)= \begin{cases}s^{\varepsilon} & \text { if } 0 \leq s \leq \lambda, \\ \lambda^{\varepsilon}+\varepsilon \lambda^{\varepsilon-1}(s-\lambda) & \text { if } s>\lambda .\end{cases}
$$

Then $H_{\lambda}(s)$ is continuously differentiable, and we note that $H_{\lambda}^{\prime}(s) \leq \varepsilon \lambda^{\varepsilon-1}$, and we define the scaling factor $\alpha=\frac{1}{\varepsilon} \lambda^{-\varepsilon+1}$; whence $\alpha H^{\prime}(u) \leq 1$ for all $u \geq 0$. In order to handle the time regularization, we define $\widetilde{H_{\lambda}}(s)=H_{\lambda}\left(s^{1 /(p-1)}\right)$. We also define the function $h_{\lambda}$ by setting

$$
h_{\lambda}(s)=\int_{0}^{s} \widetilde{H_{\lambda}}(r) d r .
$$

Let $\varphi$ be a function in $\operatorname{Lip}_{0}\left(\Omega_{T}\right)$ such that $0 \leq \varphi \leq 1$. As a nonpositive test function in $(2.3)$ we choose $\phi=-\alpha \widetilde{H}_{\lambda}\left(\left(u^{p-1}\right)_{\delta}\right) \varphi^{p}$. After handling the time regularization as in the proof of Lemma 2.1, we arrive to

$$
\begin{aligned}
& -\iota \alpha p \int_{\Omega \times\{\tau\}} h_{\lambda}\left(u^{p-1}\right) \varphi^{p} d \mu+\int_{0}^{\tau} \int_{\Omega} g_{u}^{p} d \mu d t \\
& \leq \int_{0}^{\tau} \int_{\Omega} g_{u+\alpha H_{\lambda}(u) \varphi^{p}}^{p} d \mu d t+\alpha p \int_{\tau}^{T} \int_{\Omega}\left|h_{\lambda}\left(u^{p-1}\right)\right|\left|\left(\varphi^{p}\right)_{t}\right| d \mu d t .
\end{aligned}
$$

Since $0 \leq \alpha\left|H_{\lambda}^{\prime}(u)\right| \varphi^{p} \leq 1$, as in the proof of Lemma 3.1 in [4], we can show by using the pathwise properties of upper gradients and convexity that

$$
g_{u-H_{\lambda}(u) \varphi^{p}}^{p} \leq\left(1-\alpha H_{\lambda}^{\prime}(u) \varphi^{p}\right) g_{u}^{p}+\alpha p^{p} \frac{H_{\lambda}(u)^{p}}{H_{\lambda}^{\prime}(u)^{p-1}} g_{\varphi}^{p} .
$$

From (2.14) we get the result in the same way as in Lemma 2.1, since it is easily verified that $H_{\lambda}(u)^{p} H_{\lambda}^{\prime}(u)^{1-p} \leq \varepsilon^{1-p} u^{p-1+\varepsilon}$.

We prove the following logarithmic energy estimate for positive superminimizers which covers the case $\varepsilon=p-1$ in Lemma 2.1; cp. Lemma 5.1 in [4].

Lemma 2.3. Let $u$ be a positive parabolic superminimizer. Then the inequality

$$
\begin{aligned}
& -p\left[\int_{\Omega \times\{t\}}(\log u) \varphi^{p} d \mu\right]_{t=\tau_{1}}^{\tau_{2}}+\int_{\tau_{1}}^{\tau_{2}} \int_{\Omega} g_{\log u}^{p} \varphi^{p} d \mu d t \\
& \leq\left(\frac{p}{p-1}\right)^{p} \int_{\tau_{1}}^{\tau_{2}} \int_{\Omega} g_{\phi}^{p} d \mu d t+p \int_{\tau_{1}}^{\tau_{2}} \int_{\Omega}|\log u|\left|\left(\varphi^{p}\right)_{t}\right| d \mu d t
\end{aligned}
$$

holds for every $\varphi \in \operatorname{Lip}_{0}\left(\Omega_{T}\right), 0 \leq \varphi \leq 1$, and almost every $0<\tau_{1}<\tau_{2}<T$. 
Proof. As in the preceding proofs, for $\lambda>0$ we define the function $H_{\lambda}(s)$ as

$$
H_{\lambda}(s)= \begin{cases}\lambda^{1-p}+(p-1) \lambda^{-p}(\lambda-s) & \text { if } 0 \leq s \leq \lambda, \\ s^{1-p} & \text { if } s>\lambda,\end{cases}
$$

and the scaling factor $\alpha=\frac{1}{p-1} \lambda^{p}$. Hence $\alpha\left|H^{\prime}(s)\right| \leq 1$ for all $s \geq 0$. Lastly, we define $\widetilde{H_{\lambda}}(s)=H_{\lambda}\left(s^{1 /(p-1)}\right)$ and the function $h_{\lambda}$ by setting

$$
h_{\lambda}(s)=\int_{1}^{s} \widetilde{H_{\lambda}}(r) d r .
$$

The function $h_{\lambda}(s)$ is negative for $0 \leq s<1$. The positive and the negative part are written as $h_{\lambda}(s)_{+}$and $h_{\lambda}(s)_{-}$. Let $\varphi$ be a function in $\operatorname{Lip}_{0}\left(\Omega_{T}\right)$ such that $0 \leq \varphi \leq 1$. As a nonnegative test function in $(2.3)$ we choose $\phi=\alpha \widetilde{H_{\lambda}}\left(\left(u^{p-1}\right)_{\delta}\right) \varphi^{p}$, and proceed as in the proof of Lemma 2.1. We obtain

$$
\begin{aligned}
& -p\left[\int_{\Omega \times\{t\}} h_{\lambda}\left(u^{p-1}\right) \varphi^{p} d \mu\right]_{t=\tau_{1}}^{\tau_{2}}+\int_{\tau_{1}}^{\tau_{2}} \int_{\Omega}\left|H_{\lambda}^{\prime}(u)\right| \varphi^{p} g_{u}^{p} d \mu d t \\
& \leq p^{p} \int_{\tau_{1}}^{\tau_{2}} \int_{\Omega} \frac{H_{\lambda}(u)^{p}}{\left|H_{\lambda}^{\prime}(u)\right|^{p-1}} g_{\varphi}^{p} d \mu d t+p \int_{\tau_{1}}^{\tau_{2}} \int_{\Omega}\left|h_{\lambda}\left(u^{p-1}\right)\right|\left|\left(\varphi^{p}\right)_{t}\right| d \mu d t .
\end{aligned}
$$

Consider the integrals

$$
\begin{aligned}
\int_{\Omega \times\{t\}} h_{\lambda}\left(u^{p-1}\right) \varphi^{p} d \mu & =\int_{\Omega \times\{t\}} h_{\lambda}\left(u^{p-1}\right)_{+} \varphi^{p} d \mu-\int_{\Omega \times\{t\}} h_{\lambda}\left(u^{p-1}\right)_{-} \varphi^{p} d \mu \\
& =(p-1) \int_{\Omega \times\{t\}}(\log u)_{+} \varphi^{p} d \mu-\int_{\Omega \times\{t\}} h_{\lambda}\left(u^{p-1}\right)_{-} \varphi^{p} d \mu .
\end{aligned}
$$

The first term on the right-hand side is finite for almost all $t \in\left(\tau_{1}, \tau_{2}\right)$. As for the second term, we have the monotone convergence

$$
0 \leq h_{\lambda}\left(u^{p-1}\right)_{-} \nearrow(p-1)(\log u)_{-}
$$

as $\lambda \searrow 0$. Hence we may conclude that

$$
\lim _{\lambda \rightarrow 0} \int_{\Omega \times\{t\}} h_{\lambda}\left(u^{p-1}\right) \varphi^{p} d \mu=(p-1) \int_{\Omega \times\{t\}}(\log u) \varphi^{p} d \mu .
$$

From this it is straightforward to verify that the desired estimate holds.

With these revised energy estimates at our disposal, we are able to carry out an iteration procedure similar to the one presented in [4]. We obtain, in particular, the weak Harnack inequality for positive parabolic superminimizers without the technical assumption, cp. [4, Lemma 6.1]. Finally, the following Harnack inequality for positive parabolic minimizers is obtained, cp. [4, Theorem 6.6].

Theorem 2.4. Suppose that $1<p<\infty$ and the measure $\mu$ in a geodesic metric space $X$ is doubling with doubling constant $C_{\mu}$, and the space supports a weak $(1, p)$ Poincare inequality with constants $C_{p}$ and $\Lambda$. Then a parabolic Harnack inequality is valid as follows: Let $u$ be a positive parabolic minimizer in $Q_{r} \subset \Omega_{T}$. Let $0<\delta<1$. Then

$$
\underset{\delta Q^{-}}{\operatorname{ess} \sup } u \leq C \underset{\delta Q^{+}}{\operatorname{essinf}} u,
$$

where $0<C\left(C_{\mu}, C_{p}, \Lambda, p, \delta, T\right)<\infty$. 


\section{References}

[1] BJöRN, A., and J. BJöRN: Nonlinear potential theory on metric spaces. - EMS Tracts Math. 17, Eur. Math. Soc., Zürich, 2011.

[2] Kinnunen, J. and T. KuUsi: Local behaviour of solutions to doubly nonlinear parabolic equations. - Math. Ann. 337, 2007, 705-728.

[3] Kinnunen, J., N. Marola, M. Miranda JR., and F. Paronetto: Harnack's inequality for parabolic De Giorgi classes in metric spaces. - Adv. Differential Equations 17, 2012, 801-832.

[4] Marola, N., and M. Masson: On the Harnack inequality for parabolic minimizers in metric measure spaces. - Tohoku Math. J. (2) 65, 2013, 569-589.

[5] Masson, M., and J. SilJander: Hölder regularity for parabolic De Giorgi classes in metric measure spaces. - Manuscripta Math. 142, 2013, 187-214.

[6] Wieser, W.: Parabolic $Q$-minima and minimal solutions to variational flows. - Manuscripta Math. 59, 1987, 63-107.

[7] Zноu, S.: Parabolic Q-minima and their application. - J. Partial Differential Equations 7, 1994, 289-322.

Received 23 September 2013 • Accepted 7 February 2014 